\title{
Qualitätssicherung - neue Impulse oder reloaded?
}

In einer der letzten Web-Ausgaben von Diagnostic Imaging (vom 6. Sept. 2012) wurde eine Umfrage gestartet (Poll of the week), die zum Ziel hatte, herauszufinden, welche Abteilungen ein radiologisches Qualitätssicherungsprogramm haben oder sich mit dessen Aufbau beschäftigen. Aufhänger dieser Umfrage war hier wohl die fortschreitende Anwendung und Entwicklung (und rechtlichen Problemen!) in der Teleradiologie: kommerzielle Firmen bieten mittlerweile pan-europäische teleradiologische Dienste zur Reporterstellung an, wobei der Datentransfer „cross-border“ verläuft, was einheitliche - nämlich - europäische Leit- oder Richtlinien in Punkten Datenschutz, Sicherung der Patientenprivatspäre oder der Qualität der „Auswerter“ und der beteiligten technischen Infrastruktur u. a. bedingt.

Da drängt sich vielleicht bei dem einen oder anderen die Frage auf, wie es um die Qualitätssicherung in der Sonografie bestellt ist? - Ich denke, zu dieser Thematik lassen sich einige Errungenschaften aufzählen: das interne Stufenkonzept, das eine hohe persönliche Ergebnisqualität in Theorie und Praxis sicherstellt und mittels „final teaching“ individuell abge- schlossen wird. Aber auch die gegenseitige Anerkennung der Sonografie-Ausbildung von und in unseren Schwestervereinen DEGUM-SGUM (seit 2008) zeigt, dass hier schon „cross-border“-Richtlinien erarbeitet und vorhanden sind.

Schauen wir uns weiter auf europäischer Ebene um, so haben innerhalb der EFSUMB verschiedene Komittees qualitätssichernde Aspekte zur Aufgabe: das Education and Professional Standards Committee berät u.a. die Mitgliedsgesellschaften bei der Sicherung der medizinischen (Ausbildungs- \& Diagnose-) Qualität, das ECMUS Komittee bei Fragen, die die Qualität der geräte-technischen Sicherheit und Anwendung betreffen. Aktuelle jährliche Statements, Euroson Schools zu verschiedenen Schwerpunkten oder das Euroson Course Book innerhalb des web-gestützten Educational Portal sind nur einige Resultate, die in Richtung harmonisierter europäischer Ausbildung und medizinischer Qualitätssicherung geschaffen wurden oder sich mittlerweile etabliert haben.

Die „Technical Quality Assurance Group“ der EFSUMB wird aber nun den meisten Lesern nicht bekannt sein, obwohl als Task group schon seit 2008 aktiv. Diese Gruppe hat zur Zielsetzung eine Leitlinie zu erarbeiten, die es ermöglicht, Prozeduren und Bewertungen zur gerätetechnischen Qualitätssicherung auf einheitlichem hohen Niveau durchzuführen. Diese Leitlinie ist mittlerweile fertiggestellt, vom EFSUMB Executive Board genehmigt worden und wird in Kürze in dieser Zeitschrift erscheinen.

Durch das Vorhandensein der aktuellen harmonisierten Leitlinien, europäischen Konzepte für neue Kurse gleich von Beginn an (wie z. B. bei der Notfall-Sonografie) oder den Aktivitäten innerhalb der Euroson School auf europäischer Ebene haben wir das notwendige Rüstzeug, zukünftigen Qualitätsanforderungen gerecht zu werden. Viele KollegInnen unserer ÖGUM haben hier maßgeblich neue Impulse und Anregungen auf bi-/trilateraler, aber auch auf europäischer Ebene gegeben und gesetzt, um die Qualität in der Sonografie in ihren zahlreichen Facetten zu garantieren und auszubauen. Von einem „reloading“ (nämlich alter, schon vorhandener) Aspekte kann daher also nicht gesprochen werden. Gehen wir nun daran (falls noch nicht begonnen), diese Leitlinien und Kriterien auch in unseren Alltag zu übernehmen und umzusetzen!

\section{Christian Kollmann}

